\title{
Amon: An Advanced mesh-like Optical NoC
}

DOI:

10.1109/HOTI.2015.18

Link to publication record in Manchester Research Explorer

\section{Citation for published version (APA):}

Werner, S., Navaridas Palma, J., \& Lujan, M. (2015). Amon: An Advanced mesh-like Optical NoC. In IEEE 23rd Annual Symposium on High-Performance Interconnects (HOTI) (pp. 52-59). IEEE.

https://doi.org/10.1109/HOTI.2015.18

\section{Published in:}

IEEE 23rd Annual Symposium on High-Performance Interconnects (HOTI)

\section{Citing this paper}

Please note that where the full-text provided on Manchester Research Explorer is the Author Accepted Manuscript or Proof version this may differ from the final Published version. If citing, it is advised that you check and use the publisher's definitive version.

\section{General rights}

Copyright and moral rights for the publications made accessible in the Research Explorer are retained by the authors and/or other copyright owners and it is a condition of accessing publications that users recognise and abide by the legal requirements associated with these rights.

\section{Takedown policy}

If you believe that this document breaches copyright please refer to the University of Manchester's Takedown Procedures [http://man.ac.uk/04Y6Bo] or contact uml.scholarlycommunications@manchester.ac.uk providing relevant details, so we can investigate your claim.

\section{OPEN ACCESS}




\title{
Amon: An Advanced mesh-like Optical NoC
}

\author{
Anonymized for Blind Review
}

\begin{abstract}
Optical Networks-on-chip constitute a promising approach to tackle the power wall problem present in largescale electrical NoC design. In order to enable their adoption and ensure scalability, oNoCs have to be carefully designed with power and energy consumption in mind. In this paper, we propose Amon, an all-optical NoC design based on passive microrings and Wavelength Division Multiplexing, including the switch architecture and a contention-free routing algorithm. The goal is to obtain a design that minimizes the total number of wavelengths and microrings, the wiring complexity and the diameter. An analytical comparison with state-of-the-art design proposals of all-optical NoCs shows that the proposed design can substantially improve the most important performance metrics: hop counts, chip area, energy and power consumption. Our experimental work confirms that this improvement translates into higher performance and efficiency. Finally, our design provides a tile-based structure which should facilitate VLSI integration when compared with recent ring-like solutions. In general we show it provides a more scalable solution than previous designs.
\end{abstract}

\section{INTRODUCTION}

The transition into the many-core era caused a paradigm shift from computation-centric to communication-centric computing systems. This forced the interconnection of on-chip components, commonly implemented using electrical NoCs, to become the limiting factor regarding performance and power consumption. While electrical NoCs are an effective solution for current on-chip communication requirements, it is questionable whether they can fulfill the performance requirements and tight power budgets of future many-core systems with the ever-increasing number of cores. In fact, it was previously shown that a 256 -node $2 \mathrm{D}$ mesh $\mathrm{NoC}$ in 22nm CMOS technology exceeds the allocated network power budget by an order of magnitude [1]. This shows that the total energy consumed by on-chip electronic interconnects is bound to become unacceptably high.

The emergence of integrated silicon-compatible nanophotonic technologies may provide interconnects capable of transmitting data at low latencies, high bandwidths and low power consumption [2] [3]. This is achieved by using light as the medium to transmit data, which features a nearly distance-independent power consumption on the waveguides the equivalent to the electrical wires in an optical $\mathrm{NoC}(\mathrm{oNoC})$. As opposed to electrical interconnects, in an all-optical NoCs electrical buffering and switching, which contribute most of the power budget in electrical NoCs (see, e.g. [4]), are not needed any more. Moreover, different data packets can be sent simultaneously on a single waveguide, by using Wavelength Division Multiplexing (WDN), significantly improving the network throughput. These two properties clearly outline the advantages of employing oNoCs.
Hybrid oNoCs, featuring a mixture of both electrical and optical components, have been proposed as a first step towards exploiting emerging nano-photonic technologies [5] [6]. However, they can not make use of the full potential of optical data transmission, as the electrical parts significantly increase latency and power. All-optical NoCs do not impose these limitations, but require an underlying control network $(\mathrm{CN})$ in order to avoid contention.

Next to the significant benefits of all-optical NoCs, there are a number of design challenges imposed by currently available nano-photonics that have to be tackled in order to enable their implementation.

Power Consumption: The minimization of insertion loss (IL), number of microrings, and number of wavelengths in the $\mathrm{NoC}$ is crucial as they all contribute to the oNoC's power consumption [7]. The required laser power is determined based on the maximum IL in the network, which consists of the passing-through microring loss $(0.5 \mathrm{~dB})$, passing-by microring loss $(0.005 \mathrm{~dB})$, waveguide crossings $(0.15 \mathrm{~dB})$, bending loss $\left(0.005 \mathrm{~dB} / 90^{\circ}\right)$ and propagation loss $(1.5 \mathrm{~dB} / \mathrm{cm})$ [8]. Therefore, power consumption can be reduced by minimizing IL, which is achieved by implementing a low number of microrings and waveguides crossings. The number of wavelengths used in the network also directly contributes the total power consumption as more photodetectors and modulators are required at each node.

Energy Consumption: Electrical to optical (E/O) and optical to electrical $(\mathrm{O} / \mathrm{E})$ back-end circuitry and microring heating are the main contributors to the energy consumption of an optical $\mathrm{NoC}$, being the later the dominant. Microring heating is a tuning technique that allows microrings to maintain their resonance wavelength in the presence of process- or temperature-induced resonance mismatches [9]. The energy consumed is non-data-dependent and may limit the photonic's scaling potential as it does not scale with electrical process technology. However, other tuning techniques exist, such as the electrically-assisted tuning system, which could, when properly designed, eliminate non-data-dependent tuning power completely [9].

Although the technologies to realize all-optical NoCs are already available and the first prototypes have been recently demonstrated all the way to fabrication, it is still unclear how we can manufacture production systems out of these technologies. Spidergon [10] and QuT [7] are recently proposed oNoC architectures, but both of them fail to scale particularly well according to the above challenges. Amon is an alternative, all-optical NoC design that overcomes their limitations as it targets low power and energy consumption by addressing these key issues. Our design is based on passive microring 
resonators, and is a wavelength-routed network. It therefore performs deterministic routing and avoids contention by providing different routes for each source-destination pair. The network consists of four equal meshes that are hierarchically connected, which provides a VLSI-friendly, tile-based layout.

Contributions. This paper provides the following key contributions:

- A novel tile-based all-optical NoC design targeting major challenges of low-power implementations, i.e. low number of wavelengths and microrings. We show that our design requires less microrings than state-of-the-art proposals, and features lower diameter, area and power consumption.

- A novel deterministic routing algorithm for providing efficient communication and contention-free operation.

- An analytical comparison of our design to Spidergon and QuT, that shows that it requires $45 \%$ and $30 \%$ less microrings, respectively, for network sizes of up to 256 nodes. Network diameter is much lower and scales tremendously better $-O(\sqrt{N})$ vs $O(N)$ - than the stateof-the-art architectures. This reduces the required laser power, as on average, less switching, and passing-by of microrings occur.

- The network model of our design using DSENT shows, compared to QuT, lower power consumption, photonic and waveguide area.

\section{RELATED WORK}

The integration of silicon-compatible nano-photonics for optical interconnects evoked high interest in the research community. Given that all-optical NoC design is a rather new field, only a moderate number of architectures have been proposed yet.

Hybrid NoCs, in which electrical and optical components are combined, have recently been studied [5] [6]. While they allow for an easier design approach as electrical NoC is a thoroughly studied field, the electrical components, i.e. routers and links, increase the latency and power compared to all-optical NoCs, and thus limit the achievable performance and scalability. All-optical NoCs, only consisting of optical components, have been proposed either by using electro-optic broadband ring resonators or passive microring resonators.

Electro-optic broadband ring resonators have been used numerously in recent proposals to implement all-optical NoCs [2] [11] [12] [13] [14] [15]. In these kind of NoCs, circuit-switching is used first to establish a path between a source-destination pair before the data is injected into the network. Path setup and teardown phases prior and after the data transmission affect the latency and power of the NoC. Generally, the latency drawback can be mitigated by sending large data messages; however, packet sizes in an onchip interconnect are rather small, e.g. 64-128 bytes for the transmission of a cache line.

Optical networks based on passive microring resonators consume less power as they eliminate the need of path establishment and tear-down phases by routing packets according to their wavelengths which, in turn, decreases network latency. Moreover, passive microrings are more power efficient than broadband ring resonators. Several proposals have studied microring-based NoCs [16] [17] [18] [19] [20] [10] [7].

The photonic Clos [16] is a crossbar-based topology using point-to-point connections to decrease power consumption; however, given that the number of waveguides required for its implementation scales quadratically with the number of nodes, it may only have limited scalability. R-3PO [17] is also crossbar-based, deploying a Multi-Write-Single-Read (MWSR) bus. Using crossbar-like networks have a simpler design than those using switches, but require a high number of microrings when the network size increases.

$\lambda$-Router [18] is an all-optical, contention-free network, in which all $\mathrm{N}$ nodes are connected to one $\lambda$-router, that is composed of $N / 2 \times(N-1) 4$-port switches. Next to the quadratic scaling of switches, $\lambda$ requires $\mathrm{N}$ distinct wavelength sets, leading to a high number of microrings that have to be able to respond to a rather large set of wavelengths. This may limit the scalability of this approach.

Corona [19] is an all-optical NoC using optical token ring arbitration as a control network in order to permit a node to transmit a packet. This arbitration scheme imposes a penalty in terms of latency for larger network sizes as the waiting time for a token increases with the number of nodes. The actual data network employs a MWSR bus and uses 64 wavelengths per node to modulate data, which is impractically high as the same number of rings for modulation would be required at each node. Using less wavelengths trades off performance for fewer number of microrings, and thus less energy consumption and area.

The optical Spidergon [10] is based on the Spidergon topology, which is a ring topology where, in addition to the ring, opposite nodes are connected. It uses an electrical control network, which could, however, be replaced by common optical control network solutions such as an MWSR bus. There is one distinct, but not unique, wavelength assigned to each node, that is used by others to address this node by modulating data on this wavelength. In total, their approach requires $\mathrm{N} / 2$ wavelength sets, given that it is connected to its direct neighbors, as well as the opposite node that is in N/2 hops distance.

QuT [7] is also based on a ring topology, consisting of odd and even nodes. Each even node has a cross link to both of its neighbours at N/4 distance. As they only use N/4 distinct wavelength sets, they require bypass links on each odd node in order to avoid contention. Therefore, if two source-destinationpairs send data at the same time, they will not contend as they will use different paths through the network. They propose an efficient control network based on an MWSR optical bus, and use splitters to decrease the number of waveguides required to N/16. This also reduces the number of ring modulators to $\mathrm{N} / 16$, as one waveguide is accessed by 16 nodes. In order to address a single node in the $\mathrm{CN}$, the destination address is encoded in the request packets. The most notable benefits of QuT are the low number of wavelengths, microrings and 
simple switch design, as only two different types of switches are required.

We compare Amon to the optical Spidergon and QuT designs, as they are both all-optical microring-based NoCs and perform routing based on a signal's wavelength and are the more scalable of the architectures discussed here. Evaluations results show that they can be considered as state-of-the-art solutions of efficient, low power all-optical oNoC design [7].

\section{Network ARChitecture}

In this section, we describe Amon, our power-efficient, scalable optical $\mathrm{NoC}$ design. Our goal is to obtain a network that features a low number of wavelengths, microrings and waveguides, while providing good scalability regarding all important network characteristics.

\section{A. Control Network}

We assume an underlying control network $(\mathrm{CN})$ to avoid contention by granting access to the ejection channel of a node. When a node wants to send to a certain destination, it has to send a request first. If the destination node is free, it replies with an acknowledgment, and does not answer at all otherwise. In the latter case, the source node will wait for a certain back-off time, until it sends a request again. This process is mandatory, as each node has a single ejection channel and responds to only one wavelength set. Two simultaneously sending nodes would overwrite their messages when sending at the same time. We implemented the $\mathrm{CN}$ using an MWSR bus, similar to the one described in QuT [7].

\section{B. Data Network}

Fig. 1 shows the topology of a data network containing 64 nodes. Scaling up or down is straightforward. The numbering shows how wavelengths would be assigned in this network. For 64 nodes, each submesh has 16 wavelength sets that are assigned to each node. Every node has a unique address, as shown in Fig. 2, that is used by the other nodes to address it. Passive microring resonators are used to forward data based on the modulated wavelengths. Each node has a unique wavelength set within that submesh, allowing a network with only N/4 wavelength sets. All links are bidirectional, except the blue dashed ones. In this hierarchical structure, there are three different kinds of links:

1) Mesh Links: are the regular links contained in a $2 \mathrm{D}$ mesh topology.

2) Bypass Links: are used by nodes of a submesh in case they have to send a packet to another one. E.g. if node 7 in the upper left submesh wants to send a packet to the submesh below, it sends its packet on the bypass link to its left to node 6 , which will then forward it on its bypass link, and so on. The horizontal bypass links are bidirectional, as the two horizontal boundaries are connected to other submeshes. In contrast, vertical bypass links are unidirectional, as only one boundary is connected to other submesh.

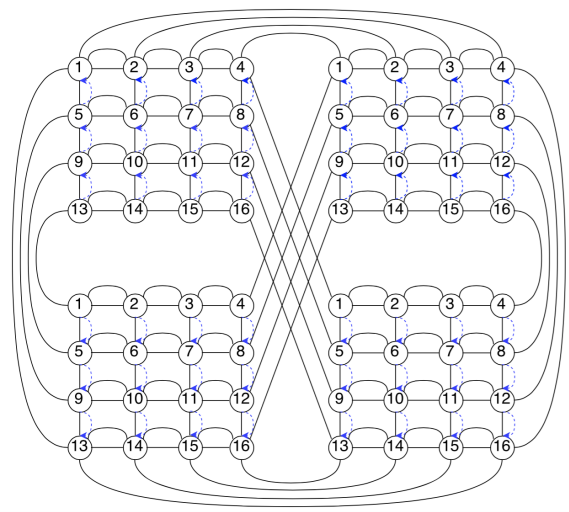

Fig. 1: Network Topology \& Wavelength Assignment

3) Inter-Mesh Links: allow data transmission between the submeshes and can essentially be treated as bypass links, as they only forward packets that either came from bypass links or were injected by the adjacent node.

Every node has a unique destination address, but shares its designated wavelengths with a node in every other submesh. Therefore, in order to address a node of another submesh, data has to be modulated on the corresponding bypass link.

\section{WDM Routing}

Routing is performed based on the positions of the source and destination:

1) In the same submesh: the packet will be injected into the local submesh and routed within using static XY routing: first advance through the $\mathrm{X}$ axis, then through the $\mathrm{Y}$ axis.

2) In a different submesh: the packet will be injected into the corresponding bypass links - e.g. from the lower left to the upper left mesh, this will use the left bypass link. Packets in the bypass will continue in that direction until they cross the inter-mesh link. Once in the desired submesh, $\mathrm{XY}$ routing is used normally, as per the above.

This simple behavior is directly implemented in hardware (see the router architecture below) so the only routing computations that need to be done are: selecting the wavelength (destination modulo $N / 4$ ) and the submesh (destination divided by $N / 4$ ). With typical power of 2 number of nodes, this can be further simplified to bit operations (the 2 MSB define the submesh and the remaining bits define the wavelength).

1) Routing Example: Fig. 2 demonstrates a few routing examples on how packets are sent through the network. It shows how the bypass and inter-mesh links are used, and how XY routing is performed. For instance, if node 43 sends to node 10 , it uses its bypass link to the left. Subsequently, packets will forwarded over their bypass links to the inter-mesh link, after which the actually mesh links in the destination mesh are used to forward the packet to 10 using $\mathrm{XY}$ routing.

In addition to correct and efficient routing, routing through an optical network with passive components must guarantee contention-free operation, i.e. if two source-destination pairs 


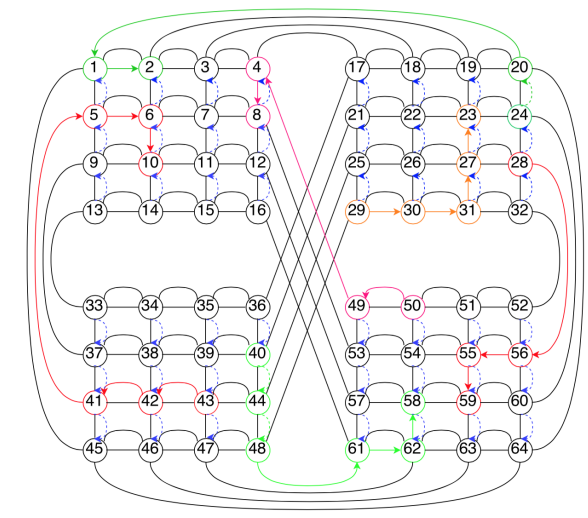

Fig. 2: Routing Examples

send on the same wavelengths, they must not use the same paths. In Amon this is achieved as follows. First, the underlying $\mathrm{CN}$ ensures that only one packet can be sent to each destination at once. Therefore, the only possibility for contention is sending data to two nodes with the same wavelength. Given that these destinations are located in different submeshes and that bypass links are physically separated, Amon design ensures the packets will never use the same waveguide.

\section{Router Microarchitecture}

All-optical NoCs using passive microring resonators can only perform predefined, deterministic routing which is implemented by employing microrings as switches. Given the different routing tasks that different nodes in our network have to perform, there are six basic different router microarchitectures in our design that feature the same types and numbers of microrings. For this description, we focus on the upper left mesh in the network in Fig. 1, and refer to nodes based on the wavelength set that is assigned to them. The other meshes have analogous architecture, and are simply mirrored regarding the inter-mesh and bypass links.

1) Nodes 1,4 (switch A): are positioned in the upper right and left corner of the mesh. They have two intra-mesh and two inter-mesh links adjacent to it.

2) Nodes 2,3 (switch B): are positioned in the top row. They are connected to three intra-mesh and one intermesh link.

3) Nodes 5,8,9,12 (switch C): are positioned in the right and left most column of the mesh. They have three intramesh and one inter-mesh link.

4) Nodes 6,7,10,11 (switch D): are positioned in the middle of the mesh, so they have four intra-mesh links but no inter-mesh links.

5) Nodes 13,16 (switch E): are positioned in the bottom right and left corner. They are connected to two intramesh and one inter-mesh link.

6) Nodes 14,15 (switch F): are positioned in the bottom row, so they are connected to three intra-mesh and no inter-mesh links.
Every switch has four injection channels. Three of them are used to send inter -mesh data, while the remaining one is used to send data within the same mesh. Add microring resonators (AmRs) are used to modulate data from the injection channel on the corresponding waveguide. As every node within the other meshes must be addressable, N/4 AmRs are required at inter-mesh injection channels. Intra-mesh injection channel do not need to generate the local wavelength and so requires $(N / 4)-1$ AmRs. Drop microring resonators (DmRs) are used to eject the data modulated on the wavelength that is assigned to the node. It thus has to be able to react to all the wavelengths within a given wavelength set. The number of Switching microring resonators (SmRs) depends on the type of switch, i.e. where it is located in the network. The more destination nodes are still reachable for a propagating packet, the more wavelength sets the microrings need to be sensible to. This, in turn, influences the number of microrings in the network, as a higher quantity of the same microring type is required to cover all possible wavelength sets. Note that we omitted the AmRs if an injection channel is directly forwarded into a certain direction. AmRs for injection channels forwarding packets to the same mesh are modulated on different waveguides. In total, there are still $(N / 4)-1$ AmRs required.

1) Switch A: Switch A (Fig. 3(a)) has to provide routing for the mesh and two inter-mesh links. In this discussion, we will describe node 1 . The connections for node 4 are the same, just mirrored. Injection channels can send data on the two intermesh links for destinations in the submesh below and to the right, on the bypass channel to the right to address the bottom right submesh, as well as on the submesh link to the right and down. Ejection must be provided for all the intra-mesh waveguides. The bypass channels, coming from the bottom and right, simply forward data as it is intended for other submeshes. Incoming data from the inter-mesh links could be intended for destinations on the right, bottom, or for switch 1 itself. Given that XY routing is performed, all destinations that are to the right of switch 1 could be addressed. As this would require a large number of microrings, we simply forward these kinds of optical data, and only perform switching when the destination is in the same column as switch 1 (performed by microring 6). Packets coming from the mesh link to the right are either intended for switch 1 itself or any of the nodes below. Therefore, according wavelengths will be send to the bottom if they are not the wavelength assigned to switch 1 (performed by microring 9). The remaining switches work in a similar fashion. Table I lists the types and their quantities required to implement this switch (if several indexes are listed, that quantity of each of them is required).

2) Switch B: Switch B (Fig. 3(b)) has to provide routing for the mesh and one inter-mesh link. Injection channels modulating data on the bypass links to the left and right are used to send data to the two submeshes below. Data meant for the submesh to the left will be modulated on the inter-mesh link, while packets intended for destinations within the same submesh could be modulated on the left, right and down link. Data that coming from the bypass links will 


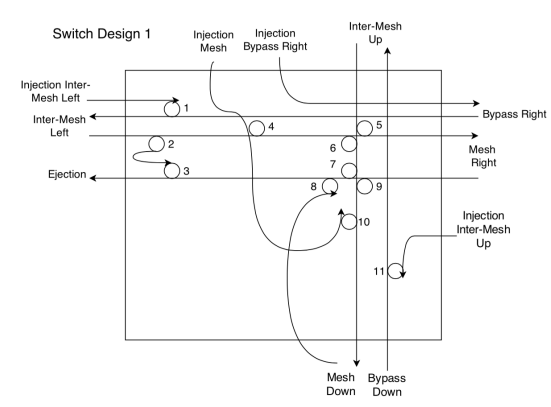

(a) Switch A

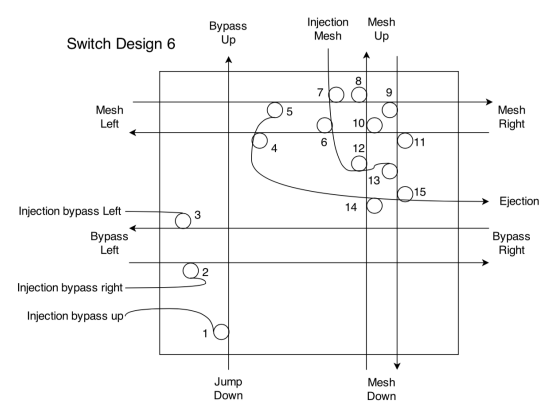

(d) Switch D

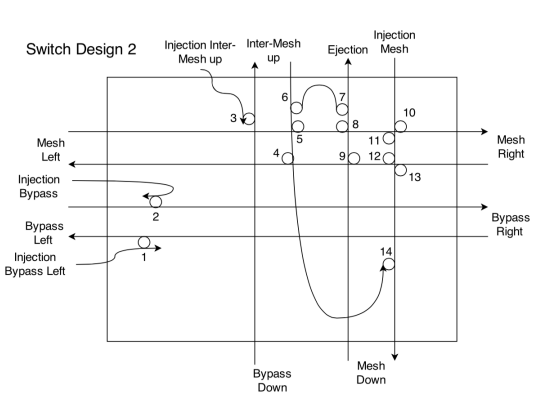

(b) Switch B

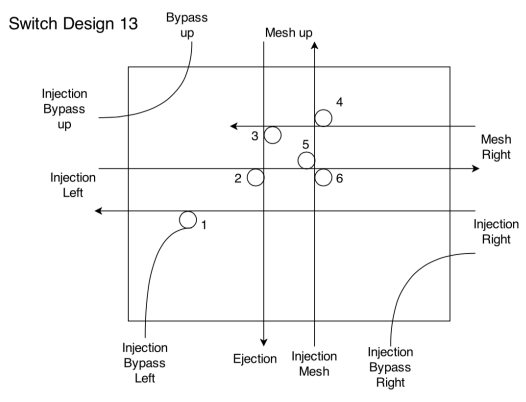

(e) Switch E

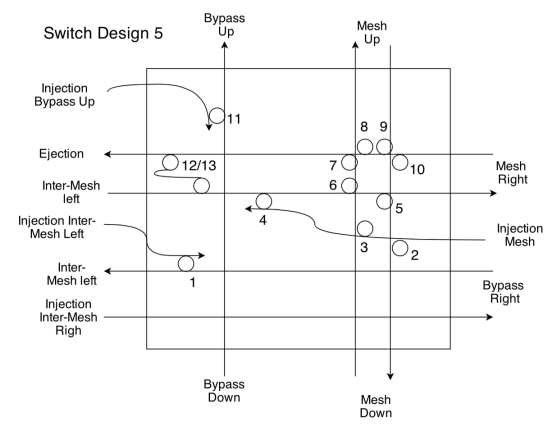

(c) Switch C

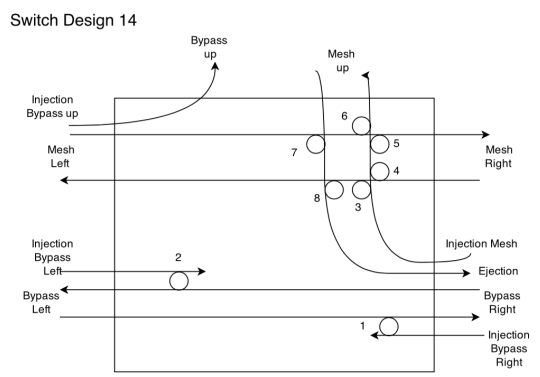

(f) Switch F

Fig. 3: All-optical switch architectures employed in our design

simply be forwarded. Packets from the inter-mesh link from the top may be addressed to any node in this submesh and, therefore, may switch to all directions in the mesh, as well as the ejection channel (performed by microrings 4,5,6, and 14). Packets incoming from the left or right will be switched down the mesh in case their wavelength conforms with one of the destination in the same column as Switch B, and will be forwarded to the opposite side otherwise. Packets coming from the bottom can only be intended for Switch 2 based on the XY routing approach, and are therefore automatically ejected. Table II lists the types and their quantities required to implement this switch. Note that some of the quantities depend on the exact position of a Switch B type router. Therefore, to have a generalized number to calculate the number of required microrings, we averaged the possible cases, e.g. for microrings 10 and 12 (inject $+X$ or $-X$ ), the aggregated number of needed wavelengths will always be $12 \lambda$, how many are needed for each will depend on the actual position in the topology. This generalization was considered in our calculations every time such a case occurred.

3) Switch C: Switch C (Fig. 3(c)) has to provide routing for the mesh and one inter-mesh link. Injection channels are assigned to the according bypass channels and intermesh channels in the same manner as in Switch $\mathrm{A} / \mathrm{B}$, i.e. in the direction of the target submesh. Data may have to be ejected from each passing waveguide except from packets coming from the adjacent bypass links, which will simply be forwarded. Incoming packets from the top or bottom submesh links will either be forwarded or ejected, as XY routing would have performed the $\mathrm{X}$ routing first. Packets from the left or right (depending the side of Switch C) will either be ejected, or switched up or down based on the modulated wavelength. If Switch $\mathrm{C}$ is the injecting node, it will modulate its data on the mesh links corresponding to the destinations address and the XY routing. Table III lists the types and quantities of microrings required to implement this switch.

4) Switch D: Switch D (Fig. 3(d)) covers every switch located in the middle of a submesh and are, therefore, the most common. Injection channels modulate their data on a waveguide in each cardinal direction for destinations in the same submesh, and on all bypass links to reach destinations in other submeshes. Data incoming from bypass links will be forwarded. Packets from the top/bottom mesh link will either be ejected if its meant for Switch D, or forwarded to its opposite direction. If packets come from the right or left, they will either be forwarded, ejected or switched up/down, based on their modulated wavelength. Table IV depicts the types of microrings and their quantities required for this switch.

5) Switch E: Switch E (Fig. 3(e)) are the switches located in the lower left and right corner of the upper two submeshes and in the upper left and right corner of the lower two submeshes. They are connected to one inter-mesh link. Data injection is performed on the bypass links and inter-mesh link to reach other submeshes, and on the mesh links up and left/right, for destinations in the same submesh. Incoming data on bypass channels will be forwarded to the opposite inter-mesh link. Incoming data from the intra-mesh link will be switched according to $\mathrm{XY}$ routing either on the mesh link up or to 
TABLE I: Microrings: Switch A

\begin{tabular}{c|c|c} 
Index & Type & Supported \# $\lambda$-sets \\
\hline \hline 1,11 & AmRs & $N / 4$ \\
4 & AmRs & $N / 4-\sqrt{N} / 2$ \\
10 & AmRs & $\sqrt{N} / 2$ \\
$2,3,7,8$ & DmRs & 1 \\
5 & SmRs & $N / 4-\sqrt{N} / 2$ \\
6 & SmRs & $\sqrt{N} / 2-1$ \\
9 & SmRs & $\sqrt{N} / 2-1$ \\
\hline \hline
\end{tabular}

TABLE II: Microrings: Switch B

\begin{tabular}{c|c|c} 
Index & Type & Supported \# $\lambda$-sets \\
\hline \hline $1,2,3$ & AmRs & $N / 4$ \\
10,12 & AmRs & $(N / 4-\sqrt{N} / 2) / 2$ \\
$6,7,8,9$ & DmRs & 1 \\
4,5 & SmRs & $N / 4-\sqrt{N} / 2$ \\
$11,13,14$ & SmRs & $\sqrt{N} / 2-1$ \\
\hline \hline \multicolumn{2}{c|}{ Total } & $N / 2+\sqrt{N} / 2+1$
\end{tabular}

TABLE III: Microrings: Switch C

\begin{tabular}{c|c|c} 
Index & Type & Supported \# $\lambda$-sets \\
\hline \hline 1,11 & AmRs & $N / 4$ \\
2,3 & AmRs & $(\sqrt{N} / 2-1) / 2$ \\
4 & AmRs & $N / 4-\sqrt{N} / 2$ \\
$7,9,12,13$ & DmRs & 1 \\
$5,6,8,10$ & SmRs & $(\sqrt{N} / 2-1) / 2$ \\
\hline \hline \multicolumn{2}{c|}{ Total } & $N / 4+\sqrt{N}+1$
\end{tabular}

the left/right. Based on XY routing, if a packet comes from the mesh link from the top, it will be ejected. Packets from the left/right will either be ejected or send up in the mesh. Cost calculations for this switch are illustrated in Table V.

6) Switch F: Switch F (Fig. 3(f)) are the switches located, depending on the submesh, in the middle of either the lowest row for upper submeshes, or the highest row for lower submeshes. They are not connected to any inter-mesh links. Data injection is performed on according bypass waveguides to other submeshes, and on the mesh links for destinations in the same submesh. Packets on the bypass waveguides will simply be forwarded. Data coming from the top mesh link will be ejected according to XY routing, and data from the left/right will be forwarded, ejected or sent up/down based on the modulated wavelength. Table VI lists the number and types of microrings required.

\section{EVALUATion}

We will first compare Amon analytically to Spidergon and QuT, considering the diameter, the number of microrings and the number of wavelength sets. Secondly, we will present the evaluation results of Amon and QuT implemented in DSENT [9] and discuss how the analytical results reflect the values extracted from DSENT. We assume a wavelength set consists of 8 wavelengths which are sent simultaneously by WDM. This increases the throughput and allows a simpler
TABLE IV: Microrings: Switch D

\begin{tabular}{c|c|c} 
Index & Type & Supported \# $\lambda$-sets \\
\hline \hline $1,2,3$ & AmRs & $N / 4$ \\
6,7 & AmRs & $(N / 4-\sqrt{N} / 2) / 2$ \\
12,13 & AmRs & $(\sqrt{N} / 2-1) / 2$ \\
$4,5,14,15$ & DmRs & 1 \\
$8,9,10,11$ & SmRs & $(\sqrt{N} / 2-1) / 2$ \\
\hline \hline \multicolumn{2}{c|}{ Total } & $N / 4+\sqrt{N} / 2+1$
\end{tabular}

TABLE V: Microrings: Switch E

\begin{tabular}{|c|c|c|}
\hline Index & Type & Supported $\# \lambda$-sets \\
\hline 1 & AmRs & $N / 4$ \\
\hline 6 & AmRs & $N / 4-\sqrt{N} / 2$ \\
\hline 2,3 & DmRs & 1 \\
\hline 4,5, & SmRs & $(\sqrt{N} / 2-1)$ \\
\hline \multicolumn{2}{|c|}{ Total } & $N / 4+\sqrt{N}-1$ \\
\hline
\end{tabular}

TABLE VI: Microrings: Switch F

\begin{tabular}{c|c|c} 
Index & Type & Supported \# $\lambda$-sets \\
\hline \hline 1,2 & AmRs & $N / 4$ \\
3,5 & AmRs & $((N / 4-\sqrt{N} / 2) / 2$ \\
7,8 & DmRs & 1 \\
4,6 & SmRs & $(\sqrt{N} / 2-1) / 2$ \\
\hline \hline
\end{tabular}

comparison to QuT and Spidergon, that assumed a similar wavelength set in their evaluation. Packets sent on the Control Network are modulated over a single wavelength, as the packet sizes are small. We used the default technology parameters for the photonic modeling [9]. We assumed an input site pitch of $1 \mathrm{e}-3$, thermal tuning with bit reshuffle as ring tuning method and a standard laser source.

\section{A. Diameter}

The network diameter in an oNoC using passive microring resonators has a different impact than in an electrical NoC. The diameter difference between our design and QuT/Spidergon would mean a tremendous performance gain in electrical NoCs, which may not be as large in oNoCs, as the bandwidth is effectively limited by the number of wavelengths used and the speed of light. Other parts of the oNoC, such as E/O and $\mathrm{O} / \mathrm{E}$ conversion, or contention resolution, have a higher impact on the performance. However, in oNoCs, a lower hop count helps to reduce energy and power. This is because of the passing-by and -through microring loss, which is a significant part of the overall power budget. A higher hop count translates into a higher number of passing-by and -through microring loss, and therefore a higher power consumption.

Both QuT and Spidergon are ring-based topologies that aim to decrease their network diameter by inserting cross links into their design. While Spidergon connects all nodes that are opposite to each other with cross links, QuT inserts cross 
TABLE VII: Network Diameter Comparison

\begin{tabular}{c|c|c|c|c} 
& Diameter & 64-Node & 256-Node & 1024-Node \\
\hline \hline Spidergon & $N / 4$ & 16 & 64 & 256 \\
QuT & $N / 4+1$ & 17 & 65 & 257 \\
Amon & $(3 \sqrt{N} / 2)-2$ & 10 & 22 & 46
\end{tabular}

TABLE VIII: Number of Microrings

\begin{tabular}{c|c|c|c|c} 
Nodes & Spidergon & QuT & Amon & Savings \\
\hline \hline 64 & 66048 & 45056 & 30336 & $54 \%, 33 \%$ \\
128 & 263000 & 170000 & 122304 & $54 \%, 29 \%$ \\
256 & 1001472 & 671744 & 498944 & $51 \%, 26 \%$
\end{tabular}

links in N/4 distance for every node with an even destination number, i.e. every second node. Given that our network is mesh-based, we result in a smaller, more scalable diameter. In fact, the longest hop count a packet will ever have to travel in our network is $3 \sqrt{N} / 2$. As illustrated in Table VII, this provides a much better scalability than N/4 and N/4 - 1 for Spidergon and QuT, respectively.

\section{B. Number of Microrings}

The number of microrings is one of the major design constraints as they are the main contributors to the overall area and power consumption. Therefore, oNoCs designs should aim to reduce the number of microrings and, in turn, the number of wavelength sets required to perform contentionfree routing. Our design requires N/4 wavelengths sets, just like QuT, which uses the lowest number of recent oNoC proposals. Spidergon uses N/2 distinct wavelength sets, which directly affects its number of microrings and scalability. The microrings are found in all the main building blocks of an oNoC, as follows:

Modulators: In order to send packets to every other node, each node has to be able to modulate data on all the required wavelengths and so, requires one modulator per injection channel and wavelength. Assuming a wavelength set size of 8 and considering that Amon has four injection channels (one per submesh) and N/4 distinct wavelength sets, the number of microrings per node is: $((N / 4) \times 8 \times 3 \times N)+((N / 4)-1) \times$ $8 \times 1 \times 1)$. The injection channel used to send data within the same mesh only needs to modulate on $(N / 4)-1$ wavelength sets as the local one is not needed.

Photodetectors: Each node requires a microring per dedicated wavelength. Considering 8 wavelengths per set, the total number of photodetectors in the network is $8 N$. The filters required prior to the photodetectors, are counted as part of the switching (see below).

Switching: The microrings required in the switches depends on the design, i.e. on the number of types of microrings in the switches and how many of them are required to respond to all the required wavelengths. We calculated the number of microrings for QuT and Spidergon with analyses similar to the one in Section 3.

Our results are listed in Table VIII and clearly show that Spidergon requires the highest number of microrings, mainly due to the larger number of wavelength sets $(\mathrm{N} / 2)$ in the network. The high number of microrings in QuT is explained by the switch design. Many of the microrings need to respond to N/4 wavelength sets, whereas Amon mesh structure allows more sophisticated and fine-tuned types of microrings, as they only need to respond to a lower number of wavelengths. The percentage of microring savings, listed in Table VIII, slightly decrease with a higher node count. However, for 256 Nodes, the savings are still at $51 \%$ and $26 \%$ compared to Spidergon and QuT, respectively.

These theoretical results could be confirmed by means of a practical evaluation using DSENT. Table IX lists the power results of Amon and QuT for 64, 144, and 256 nodes. For simplicity, we chose these sizes as they allow for square submeshes in Amon. Note, however, that non-square submeshes are perfectly possible in Amon.

TABLE IX: Power Comparisons for 64, 144 and 256 Nodes

\begin{tabular}{c|c|c|c|c|c|c} 
& \multicolumn{2}{|c}{64 Nodes } & \multicolumn{2}{c}{144 Nodes } & \multicolumn{2}{c}{256 Nodes } \\
Power $(\mathrm{W})$ & QuT & Amon & QuT & Amon & QuT & Amon \\
\hline Laser & 2.27 & 1.71 & 11.59 & 9.19 & 36.99 & 32.36 \\
Ring Heater & 16.5 & 16.2 & 82.45 & 82.89 & 261.43 & 260.57 \\
\hline \hline Total & 18.77 & 17.91 & 94.48 & 92.08 & 298.42 & 292.93
\end{tabular}

The photonic area results obtained from DSENT are listed in Table X, and comprise the area of modulators, detectors and switching rings, shows that Amon can reduce chip area. This is as expected based on the previous analytical results.

\section{Wiring}

While it is difficult to assert the wiring complexity quantitatively for oNoCs, we can observe that both Spidergon and QuT decrease their diameter by introducing cross links. These cross links possibly lead to a large number of waveguide crossings, particularly with an increasing network size. Our proposal, on the other hand, features very few crossings caused by the topology. The only obvious crossing is the one of the intermesh links in the middle of the topology. However, a wire only crosses $\sqrt{N} / 2$ other wires, which is much less than in Spidergon and QuT, where every node or every second node is connected to its opposite counterpart, respectively. Given the insertion loss caused by waveguide crossings, this is another benefit in terms of power efficiency.

Considering the number of waveguides required in these networks, we can make interesting observations, too. The number of cross-links in Spidergon and QuT scales with $N / 2$, which means that the number of cross-links that have to be added increases linearly. In order to scale our proposal up, however, only inter-mesh/bypass links have to be added, which scale with $6 \times \sqrt{N} / 2$. Mesh waveguides only need to be made longer to add further nodes to a mesh. Therefore, we may feature a lower wiring complexity and number of waveguides, further simplifying implementation. The meshlike, tile-based structure of our network further facilitates VLSI implementation. The results obtained through DSENT (Table XI) confirm our theoretical assumptions, as they show 
TABLE X: Photonic Area in $m^{2}$

\begin{tabular}{c|c|c|c} 
\#Nodes & QuT & Amon & Savings \\
\hline \hline 64 & $1.16 \mathrm{e}-04$ & $1.05 \mathrm{e}-04$ & $10 \%$ \\
144 & $1.24 \mathrm{e}-03$ & $1.18 \mathrm{e}-03$ & $5 \%$ \\
256 & $7.03 \mathrm{e}-03$ & $6.55 \mathrm{e}-03$ & $7 \%$
\end{tabular}

TABLE XI: Total Waveguide Area in $m$

\begin{tabular}{c|c|c|c} 
Network & QuT & Amon & Savings \\
\hline \hline 64 & $4.8 \mathrm{e}-06$ & $2.4 \mathrm{e}-06$ & $100 \%$ \\
144 & $1.4 \mathrm{e}-05$ & $7.9 \mathrm{e}-06$ & $77 \%$ \\
256 & $3.1 \mathrm{e}-05$ & $1.9 \mathrm{e}-05$ & $63 \%$
\end{tabular}

that QuT requires significantly more waveguide resources than Amon.

\section{Discussion}

We the number of microrings and diameter sizes in Spidergon, QuT and Amon, and showed that the latter is more energy efficient and more scalable. The lower diameter may, next to a higher energy efficiency, also lead to better performance metrics. Our theoretical results were confirmed by modeling the networks in DSENT. Given that previous designs were ring-based topologies, our mesh-based design provides a structure that is well-suited for VLSI implementation of the tilebased layout of manycore systems. Moreover, the mesh-based topology features a lower number of waveguides as well as less waveguide crossings, with the subsequent savings in power and area. The benefit of QuT and Spidergon is their simple implementation, as QuT only has two different router designs and Spidergon only one. Amon features in total six different designs, and may therefore be more complex to implement. However this enables fine-grained wavelength selection which, in turn, reduces the number of microrings promoting further savings in area and laser power. It could be observed that the ring tuning power is significantly higher than the laser power. We intend to use other ring tuning methods in future work to find the most suitable one.

\section{CONCLUSION}

On-chip optical interconnection networks provide a wide range of design possibilities to enable future, low power and high performance many-core systems. However, the integration of current photonic technologies in NoCs requires sophisticated designs that allow low power operation. We proposed Amon, an oNoC that achieves low power operation by minimizing the number of microrings, hop count and waveguide crossings. Analytical results regarding these metrics were presented and confirmed by using DSENT. The mesh-based topology facilitates the tile-based VLSI implementation and decreases wiring compared to previous ringbased topologies. Future work includes the evaluation of the performance results under realistic traffic patterns, so that the impact of the low diameter on energy-per-bit and throughputper-watt characteristics can be assessed. We intend to integrate our DSENT model into the Graphite simulator [21] for that purpose.

\section{ACKNOWLEDGMENTS}

Anonymized for blind review

\section{REFERENCES}

[1] J. Owens et al., "Research challenges for on-chip interconnection networks," Micro, vol. 27, no. 5, pp. 96-108, 2007.

[2] A. Shacham et al., "Photonic networks-on-chip for future generations of chip multiprocessors," IEEE Transactions on Computers, vol. 57, no. 9, pp. 1246-1260, 2008.

[3] J. W. Goodman, F. J. Leonberger, S.-Y. Kung, and R. A. Athale, "Optical interconnections for vlsi systems," Proceedings of the IEEE, vol. 72, no. 7, pp. 850-866, 1984.

[4] Y. Hoskote, S. Vangal, A. Singh, N. Borkar, and S. Borkar, "A 5-ghz mesh interconnect for a teraflops processor," Micro, IEEE, vol. 27, no. 5, pp. 51-61, Sept 2007.

[5] C. Li et al., "Luminoc: A power-efficient, high-performance, photonic network-on-chip for future parallel architectures," in PACT, 2012. ACM, 2012, pp. 421-422.

[6] Y. Pan et al., "Firefly: illuminating future network-on-chip with nanophotonics," in ACM SIGARCH Computer Architecture News, vol. 37, no. 3. ACM, 2009, pp. 429-440.

[7] P. K. Hamedani, N. E. Jerger, and S. Hessabi, "Qut: A low-power optical network-on-chip," in NOCS, 2014. IEEE, 2014, pp. 80-87.

[8] K. Bergman et al., Photonic Network-on-Chip Design. Springer, 2014.

[9] C. Sun et al., "Dsent-a tool connecting emerging photonics with electronics for opto-electronic networks-on-chip modeling," in NOCS, 2012. IEEE, 2012, pp. 201-210.

[10] S. Koohi and S. Hessabi, "Scalable architecture for a contention-free optical network on-chip," Journal of Parallel and Distributed Computing, vol. 72, no. 11, pp. 1493-1506, 2012.

[11] J. Chan and K. Bergman, "Photonic interconnection network architectures using wavelength-selective spatial routing for chip-scale communications," Journal of Optical Communications and Networking, vol. 4, no. 3, pp. 189-201, 2012.

[12] I. Artundo et al., "Low-power reconfigurable network architecture for on-chip photonic interconnects," in HOTI, 2009. IEEE, 2009, pp. 163169.

[13] J. Chan et al., "Architectural exploration of chip-scale photonic interconnection network designs using physical-layer analysis," Journal of Lightwave Technology, vol. 28, no. 9, pp. 1305-1315, 2010.

[14] H. Gu et al., "A low-power fat tree-based optical network-on-chip for multiprocessor system-on-chip," in DATE, 2009. European Design and Automation Association, 2009, pp. 3-8.

[15] G. Hendry et al., "Analysis of photonic networks for a chip multiprocessor using scientific applications," in NOCS, 2009. IEEE, 2009, pp. 104-113.

[16] A. Joshi et al., "Silicon-photonic clos networks for global on-chip communication," in NOCS, 2009. IEEE Computer Society, 2009, pp. 124-133.

[17] R. Morris et al., "Dynamic reconfiguration of 3d photonic networkson-chip for maximizing performance and improving fault tolerance," in MICRO, 2012. IEEE Computer Society, 2012, pp. 282-293.

[18] M. Briere et al., "System level assessment of an optical noc in an mpsoc platform," in DATE, 2007. EDA Consortium, 2007.

[19] D. Vantrease et al., "Corona: System implications of emerging nanophotonic technology," in ACM SIGARCH Computer Architecture News, vol. 36, no. 3. IEEE Computer Society, 2008, pp. 153-164.

[20] M. J. Cianchetti et al., "Phastlane: a rapid transit optical routing network," in ACM SIGARCH Computer Architecture News, vol. 37, no. 3. ACM, 2009, pp. 441-450.

[21] J. E. Miller, H. Kasture, G. Kurian, C. Gruenwald, N. Beckmann, C. Celio, J. Eastep, and A. Agarwal, "Graphite: A distributed parallel simulator for multicores," in High Performance Computer Architecture (HPCA), 2010 IEEE 16th International Symposium on. IEEE, 2010, pp. 1-12. 